\title{
Enseñanza de la cultura emprendedora en la universidad: El caso de la Universidad Austral de Chile
}

\author{
Cristian M. Colther ${ }^{1^{\star}}$, Ester Fecci ${ }^{2}$, Gloria Cayun ${ }^{3}$ y Julio Rojas-Mora ${ }^{4}$ \\ (1) Instituto de Economía, Universidad Austral de Chile, calle Viel s/n, Isla Teja, Valdivia-Chile. \\ (correo-e: cristiancolther@uach.cl) \\ (2) Instituto de Administración, Universidad Austral de Chile, calle Viel s/n, Isla Teja, Valdivia-Chile. \\ (correo-e: efecci@uach.cl) \\ (3) Programa CEM, Facultad de Cs. Económicas y Administrativas, Universidad Austral de Chile. Valdivia-Chile. \\ (correo-e: gloria.cayun@uach.cl) \\ (4) Departamento de Ing. Informática, Universidad Católica de Temuco, Temuco-Chile. (correo-e: jrojas@inf.uct.cl) \\ * Autor a quien debe ser dirigida la correspondencia.
}

Recibido Dic. 4, 2019; Aceptado Ene. 31, 2020; Versión final Mar. 31, 2020, Publicado Ago. 2020

\begin{abstract}
Resumen
Este artículo tiene como objetivo presentar la experiencia de la Facultad de Ciencias Económicas y Administrativas de la Universidad Austral de Chile en implementar un programa que fomenta el emprendimiento y el desarrollo de habilidades para emprender entre sus estudiantes de pregrado. Como metodología, el programa ha utilizado métodos de reciente uso en el contexto universitario a través de la creación de una asignatura que facilita el desarrollo de proyectos de emprendimiento con un enfoque social, colaborativo e interdisciplinario. De los resultados de la experiencia, se menciona la generación de 39 proyectos estudiantiles en el período de análisis entre otras iniciativas realizadas por el programa. Se concluye que la experiencia puede aportar a la discusión y diseño de este tipo de experiencias en las universidades chilenas.
\end{abstract}

Palabras clave: emprendimiento; educación universitaria; innovación docente; educación empresarial

\section{Teaching of entrepreneurial culture at the university: the case of the University Austral de Chile}

\begin{abstract}
This paper aims to present the experience of the Faculty of Economics and Administration Science at the University Austral of Chile on implementing a program that encourages entrepreneurship and the development of skills in undergraduate students. The methodology of the program employs the university context by creating an academic subject that facilitates the development of entrepreneurship projects having a social, collaborative, and interdisciplinary approach. The generation of 39 student projects in the analysis period are examined among other initiatives performed in the program. In conclusion, the program implemented at the Faculty of Economics and Administration Science at the Austral of Chile University can contribute to the discussion and design of this type of experience at other Chilean universities.
\end{abstract}




\section{INTRODUCCIÓN}

En los últimos años, en la sociedad ha surgido una creciente demanda hacia las universidades para que asuman nuevas tareas, más allá de las tradicionales actividades de investigación y enseñanza, vinculadas principalmente con el desarrollo económico local y la generación de innovación en los territorios (Wong et al., 2007), también que sean instituciones más cercana a las problemáticas y necesidades de la sociedad, saliendo de la torre de marfil (Etzkowitz et al., 2000), y demandando que la investigación y el nuevo conocimiento se oriente a un contexto aplicado, que aporte con soluciones a las complejas problemáticas de la sociedad y genere innovación que agregue valor a los bienes y servicios de la economía, y permita la creación de nuevas actividades y empleos (Heaton et al. 2019).

En este contexto, una de las tareas nuevas que las universidades deben asumir es el estimulo del emprendimiento entre sus estudiantes y académicos, que implica asumir un rol activo por parte de la universidad, para generar capacidades y destinar recursos para estimular la creación de ideas de negocios y el valor del autoempleo desde la academia (Clark, 1998; Grigg, 1994; Heaton et al., 2019). Esta nueva tarea se ha conceptualizado en el término en inglés entrepreneurial university o "universidad emprendedora", es decir, una universidad que estimula el emprendimiento entre sus estudiantes, y que mediante unidades especializadas en metodologías y conocimientos específicos, facilitan el desarrollo de habilidades y competencias en los estudiantes, para la generación de ideas de negocios con un fuerte enfoque en lo económico y social (Zhang et al., 2014, Freel et al., 2019). Sin embargo, un entorno favorable y recursos financieros para crear nuevos negocios no garantizan el surgimiento de empresas, y mucho menos la generación de ideas innovadoras. Aunque existen modelos relacionados con el surgimiento de empresas centradas en universidades como Stanford o el Instituto de Tecnología de Massachusetts, estos modelos no son fáciles de replicar y requieren una mirada contextualizada del entorno, así como un cambio cultural dentro de la universidad (Etzkowitz y Zhou, 2008).

La cultura empresarial se está volviendo cada vez más influyente en la educación superior a nivel mundial, y es un concepto que varios autores han explorado en los últimos años (Etzkowitz, 2004; Marzocchi et al., 2019). Esto producto de la necesidad por parte de las empresas y la sociedad de que los estudiantes desarrollen habilidades empresariales; sea para desempeñar tareas más complejas en las empresas o facilitar la externalización de servicios, estimulando el autoempleo entre sus empleados en el caso de las empresas; o producto de la masificación de la educación superior y la incertidumbre de los mercado laborales, que plantean la necesidad de generar profesionales capaces de innovar y autoemplearse (Gibb et al., 2013). En este contexto, el emprendimiento se ha convertido en un rol emergente que las universidades deben asumir para facilitar la transferencia de tecnología y el desarrollo de la ciencia aplicada (Smilor et al.,1993) y reforzar su función social (Grigg, 1994). Por tanto, parte de la formación universitaria moderna debe considerar fomentar la creatividad y la capacidad de respuesta al cambio, dado que se requiere creatividad para que haya innovación, y con esta el emprendimiento. Por estos motivos, es necesario que las universidades fomenten una cultura del emprendimiento para responder a las diversas aspiraciones de los estudiantes, que a su vez, pueden usar sus habilidades para responder a las necesidades de la sociedad (Clark, 1998).

Desde una perspectiva global, otros autores como Etzkowitz et al. (2000) menciona que la universidad se convierte en un elemento clave del sistema de innovación, en el contexto de una economía basada en el conocimiento, tanto como proveedor de capital humano avanzado, como un semillero de nuevas empresas, y por tanto, una estimuladora del emprendimiento. Por su parte, Lazzeroni y Piccaluga (2003) proponen cuatro funciones a desarrollar por las universidades, las tradicionales como son la generación de conocimiento, formación de capital humano, y las nuevas como son la transferencia de tecnología y el impulsar el desarrollo local; y en esta última, el emprendimiento puede jugar un rol fundamental. Audretsch (2014) va más allá y señala que el papel de la universidad en la sociedad es fundamental para proporcionar actividades de pensamiento, liderazgo y emprendimiento para mejorar el capital humano. Su objetivo es garantizar que las personas prosperen en la sociedad; Una misión más amplia que solo la comercialización de la investigación universitaria y la generación de nuevas empresas o nuevos negocios.

Sin embargo, para que la universidad pueda generar espacios para la formación del emprendimiento, deben tener la capacidad de flexibilizar su funcionamiento, y disponer de elementos que faciliten la transformación organizacional para implementar programas que se alejan de la docencia tradicional; además de desarrollar vínculos con organizaciones privadas y públicas, y capacidad de captar fuentes alternativas de financiamiento para apoyar y estimular las actividades empresariales que puedan nacer en su interior. En este aspecto, algunos autores mencionan que la universidad que fomenta el enfoque empresarial debe ser proactiva, caracterizada por su dinamismo e interactividad con la sociedad; y es influyente e influida por el medio, capaz de armonizar los asuntos científicos con las habilidades de gestión, y se caracteriza por la transferencia sistemática de conocimiento a través de la tecnología, la capacitación y la adaptación continua a las demandas del mercado laboral (Clark, 2004; Zaharia y Gibert, 2005). 
Este artículo tiene por objetivo presentar la experiencia de la Facultad de Cs. Económicas y Administrativas de la Universidad Austral de Chile en implementar un programa que fomenta el emprendimiento y el desarrollo de habilidades para emprender entre sus estudiantes de pregrado en el período 2015-2018. Utilizando métodos de reciente uso en el contexto universitario, a través de la creación de una asignatura que, facilita el desarrollo de proyectos de emprendimiento con un enfoque social, colaborativo e interdisciplinario. La experiencia puede aportar a la discusión y diseño de este tipo de experiencias en las universidades chilenas.

\section{OTROS ANTECEDENTES}

Para que las universidades tradicionales sean capaces de fomentar el emprendimiento, además de una arquitectura institucional flexible, como se mencionó anteriormente, se deben desarrollar vínculos con las empresas a través de sus académicos y centros, que faciliten la difusión de la innovación y la determinación de las habilidades que requieren las empresas, y se recomienda una apertura a redes internacionales que estimulen la investigación y la implementación de proyectos colaborativos. Sin embargo, esto no es simple de hacer, y tampoco existen recetas estándares que se puedan aplicar, con lo cual las universidades tradicionales deben adaptar su estructura y formas de gestión, y crear diferentes formas de vínculos con su entorno.

Ejemplos de vínculos se puede encontrar en Martinelli et al. (2008) que realizaron un estudio en una universidad del Reino Unido sobre el tipo de vínculos que pueden existir entre la universidad y su entorno, y propusieron una tipología de vínculos externos. Algunos de ellos son: regalías de patentes, consultoría, investigación colaborativa, acuerdos o contratos de investigación, patentes y licencias de investigación de estudiantes, creación de una empresa y un esquema de transferencia de conocimiento, entre otros posibles. Estos vínculos posibilitan que los académicos y alumnos familiarizados con una cultura emprendedora, puedan desarrollar o articular ideas y conectar la universidad con su entorno, facilitando el acceso a recursos financieros y el desarrollo de proyectos que satisfacen las necesidades del entorno.

Otros autores agregan a estos vínculos ampliando el espectro de tipos de empresas que se pueden crear y características que trascienden el ámbito de la investigación y la enseñanza que las universidades deben desarrollar como son: a) interacción: interactúa estrechamente con la industria y el gobierno, b) independencia: es una institución relativamente independiente; no depende de otra esfera institucional, c) hibridación: hace que su arquitectura organizacional sea flexible para resolver las tensiones entre los principios de interacción e independencia, y de ese modo cumple ambos objetivos simultáneamente, y d) reciprocidad: se actualiza continuamente su estructura interna y su relación con la industria y el gobierno (Etzkowitz, 2013).

La formación en emprendimiento en las universidades requiere además de otros elementos que faciliten y protejan la innovación generada por los emprendedores, como son actividades y políticas de transferencia de conocimiento y tecnología, infraestructura y recursos financieros asociados a la generación de nuevas empresas (parques, incubadoras, fondos de financiamiento), disponibilidad de programas de capacitación para instructores, enlaces con programas de pregrado y postgrado en su currículo formativo; con lo que el emprendimiento, se convierte casi en un concepto interdisciplinar, intrínseco al desarrollo de todos los estudiantes y el personal docente universitario, que se aleja del modelo de escuela de negocios convencional (Gibb et al., 2013). Por lo mencionado anteriormente, la formación en emprendimiento no deja de ser un importante nuevo desafío que las universidades a nivel internacional han asumido en estas últimas décadas, y de forma muy reciente en el contexto latinoamericano.

En el caso de Chile existen algunos estudios que muestran que las universidades tienen una preocupación por el. Por ejemplo, Herrera y Villalobos (2018) muestra evidencia de que las universidades chilenas han realizado esfuerzos para implementar iniciativas de formación en emprendimiento, considerando recursos; políticas y normativas; estructuras organizativas; reconocimientos e incentivos; instancias para socializar emprendimientos; y soporte curricular e instrumentos de diagnóstico y evaluación (ver por ejemplo una propuesta en Soria-Barreto et al., 2016). Sin embargo, los autores mencionan que los resultados de estas iniciativas presentan una heterogeneidad en su implementación y logros; y por tanto se observa que la formación en emprendimiento a nivel de pregrado en las universidades todavía es incipiente y sin un desarrollo o cuerpo común, respecto de los elementos formativos y de su estructura organizacional que son necesarias para su desarrollo.

Respecto de las metodologías que pueden utilizarse para estimular el emprendimiento en las universidades, existe diversidad y varias experiencias exitosas a nivel internacional (Herrera y Villalobos, 2018; Zhang et al., 2014); pero dadas sus características particulares respecto del grado de desarrollo de las universidades que las implementan, las diferencias culturales o disponibilidad de recursos públicos y privados que disponen en su entorno local o nacional, en general son de difícil replicación en Chile. Por estos motivos, se percibe como un campo interesante de desarrollo por parte de las universidades, el definir el tipo de acciones, los métodos 
y los instrumentos que pueden ser útiles para promover en los estudiantes el emprendimiento. A continuación, presentamos la experiencia desarrollada por la Universidad Austral de Chile, considerada como una universidad tradicional en el contexto del sistema universitario chileno (Mondaca et al. 2019) y algunos de sus resultados.

\section{EL PROGRAMA CENTRO DE EMPRENDIZAJE}

En esta sección se presenta la experiencia de un programa implementado en la Facultad de Ciencias Económicas y Administrativas de la Universidad Austral de Chile (UACh), que busca introducir en la formación tradicional nuevos elementos formativos vinculados con la formación en competencias y habilidades para el emprendimiento. Comenzamos el relato presentando a la Universidad y un breve contexto, para luego desarrollar la experiencia desde su génesis, las metodologías utilizadas y organización, y una breve descripción de las acciones realizadas y sus resultados.

\section{Contexto de la Universidad Austral de Chile}

La Universidad Austral de Chile (UACh), es una de las seis universidades tradicionales más importantes de Chile, que en forma autónoma ha incidido en el desarrollo socioeconómico del sur austral de Chile, creada el año 1954, es uno de los centros educativos más grandes del sur de Chile, inicialmente enfocado en las ciencias básicas vinculadas a la agricultura, silvicultura y medicina veterinaria, hoy presenta una variada oferta formativa. La UACh ha desempeñado un papel importante en el desarrollo de la región en los últimos 65 años, formando capital humano avanzado e impulsando la investigación y el desarrollo en las actividades relevantes de las economías en las regiones del sur de Chile, como son las actividades silvoagropecuarias, la industria de los alimentos lácteos y la salmonicultura. La Universidad presenta sedes en las ciudades de Valdivia, Osorno, Puerto Montt, Coyhaique, y actualmente dicta 70 carreras de pregrado, 37 programas de Magíster y 12 programas de Doctorado, que implica poner a disposición de la comunidad una diversa oferta formativa de pre y postgrado en la macrozona sur austral de Chile. Actualmente se encuentra acreditada por la Comisión Nacional de Acreditación (CNA-Chile) por un período de 6 años (de un máximo de 7), desde noviembre de 2015 hasta noviembre de 2021 y figura en posiciones destacadas en ranking nacionales e internacionales, debido a su calidad académica en pre y postgrado, y a su producción científica que la localiza dentro de una de las 5 universidades más productivas del país (Mondaca et al., 2019).

\section{Creación y evolución del CEM}

EI CEM nace como iniciativa de un grupo de académicas a principios del dos mil, que buscaban desarrollar una iniciativa para promover el espíritu emprendedor y el conocimiento útil para desarrollar proyectos colaborativos con impacto local, responsabilidad social y conciencia ambiental dado las problemáticas de informalidad y pobreza presentes en la región de Los Ríos. Además de la necesidad de generar un espacio de reflexión en la Facultad de Cs. Económicas y Administrativas, que vincule a los alumnos con la comunidad local, el mundo empresarial y las organizaciones sociales. En forma paralela, Servicios Públicos del sur de Chile, vinculados al fomento productivo planteaban la necesidad de involucrar a la Universidad como actor relevante, para colaborar con su experticia técnica, y a sus estudiantes para dar soluciones a las problemáticas mencionadas anteriormente. Estas conversaciones posibilitan que el año 2007 se suscriba un convenio entre un organismo gubernamental y la carrera de Ingeniería Comercial para desarrollar talleres de consultoría de empresas, en donde los alumnos de último año de la carrera de Ingeniería Comercial asesoran a pequeñas empresas en sus negocios y estrategias de formalización, las que se realizan hasta hoy en día.

La experiencia acumulada relacionada con el convenio facilita la creación del programa denominado "Centro de Emprendizaje-CEM" el año 2014, radicando el programa en la Facultad de Cs. Económicas y Administrativas de la Universidad, como una iniciativa de acercamiento a la lógica de diseños de programas colaborativos a nivel de alumnos de pregrado que promueve el autoempleo a través del emprendimiento. Esta iniciativa, es financiada primero por la Corporación de Fomento de la Producción (CORFO) y luego por la Iniciativa de Participación Económica Juvenil (YEPI, sigla en inglés de Youth Economic Participation Initiative https://talloiresnetwork.tufts.edu/the-youth-economic-participation-initiative/), un programa internacional impulsado por la red de colaboración universitaria The Talloires Network (https://talloiresnetwork.tufts.edu/) para promover el cambio en la forma en que las universidades de todo el mundo preparan a sus estudiantes para la vida laboral después de graduarse.

Esta iniciativa, aprovechando la estructura, los recursos y los vínculos de la Universidad con el medio local, comienza a potenciar proyectos de desarrollo comunitarios trabajando con agrupaciones juveniles y microempresarios formales e informales, incorporando la formación de habilidades relacionales y formación en emprendimiento. Que finalmente culmina con la creación de una asignatura optativa denominada "Emprendizaje colaborativo", la cual vincula a los y las estudiantes de la universidad con sus intereses de 
desarrollo personal, integrando metodologías disruptivas que facilitan un aprendizaje significativo, el desarrollo de una actitud colaborativa y la capacidad de generar ideas de negocios y proyectos, estimulando el trabajo interdisciplinario entre alumnos de diferentes carreras de la Universidad. Esta nueva asignatura, tiene como objetivo adicional, desarrollar en los alumnos, un vinculo con el medio local, con la comunidad y con el cuidado ambiental, dimensiones que son transversales en la formación integral de los estudiante y que aporta a desarrollar las competencias sello de la Universidad vinculadas a demostrar compromiso con el conocimiento, la calidad, la excelencia, la libertad, la naturaleza y el desarrollo sustentable, el respeto por la diversidad y actuar con responsabilidad social (UACh).

Además, la asignatura busca el desarrollo de habilidades para trabajar en forma autónoma y colaborativa, a través de metodologías lúdicas, que exploran las vocaciones e intereses de los estudiantes y entregan herramientas básicas de planificación financiera; acompañando en forma sistemática a los alumnos en el diseño de la idea de negocio o prototipo de nuevos bienes o servicios, y desarrollando en forma progresiva durante el transcurso del semestre un proyecto o idea de negocio. Con este tipo de trabajo se prepara a los estudiantes para su futura vida laboral, sea como asalariado, profesional independiente o emprendedor que se autoemplea, en línea con los esfuerzos más recientes para promover el emprendimiento, la innovación y el autoempleo como una estrategia nacional de desarrollo económico (Mandakovic et al., 2015).

\section{Metodologías para la enseñanza del emprendimiento}

Parte de la estrategia de desarrollo del CEM ha sido formar estudiantes con nuevas metodologías para estimular el espíritu emprendedor y ciclos formativos de los estudiantes, y poner a disposición de los académicos nuevas metodologías que pueden enriquecer los procesos de aprendizaje de los estudiantes. El CEM utiliza diversas metodologías para estimular y/o desarrollar el espíritu y una actitud emprendedora en los estudiantes de pregrado, que van desde entregar conocimientos que facilitan el diseño y la planificación de un negocio, hasta aquellas que facilitan un proceso formativo de calidad, en términos de significado, profundidad y estímulo para la reflexión crítica; fuertemente fundado en la participación (Disterheft et al., 2016), que incluyen temas como: a) facilitación b) comunicación y principios democráticos, c) la calidad de la colaboración, d) las relaciones existentes entre el ser humano y la naturaleza. Los métodos pueden ser variados, como por ejemplo, técnicas de facilitación y trabajo grupal, o enfoques emergentes como la teoría de sistemas, ó enfoques que se centran en el aprendizaje transformador y el liderazgo como una forma de facilitación, como son: la Teoría U (Scharmer, 2016); Dragon Dreaming; Art of Hosting; World Cafe, entre muchos otros métodos disponibles (Disterheft et al., 2015).

Estos métodos que a menudo se aplicaban en proyectos comunitarios u organizaciones e instituciones locales, han permeado cada vez más en las universidades. Las metodologías mencionadas anteriormente facilitan el aprendizaje experiencial para el desarrollo de ideas de proyectos colaborativos entre los alumnos. Además, se genera sesiones denominadas Circulo de apoyo, en donde se desarrollan procesos de aprendizaje colaborativo, a través de espacios de encuentro para que grupos de docentes, empresarios y estudiantes aprendan juntos, con el objetivo de generar inteligencia colectiva que active ideas de proyectos en los estudiantes.

De las metodologías no tradicionales y bastante recientes se pueden mencionar la "Teoría U" que es un método de desarrollo personal que se enfoca en fomentar la innovación en las personas a través de 7 pasos que facilitan el autoconocimiento y gestión personal que facilita el desarrollo de proyectos en colaboración (Scharmer, 2016). El Dragon Dreaming que se puede definir como una metodología que enseña un marco de planificación estratégica con un conjunto de técnicas utilizadas para desarrollar proyectos colaborativos y con enfoque social, y de sostenibilidad ambiental. Está construido sobre los pilares del crecimiento personal, la construcción de la comunidad y el servicio a la tierra, y enfatiza un ciclo de soñar, planificar, hacer y celebrar. Señala que compartir y poner a disposición su sueño con otros es vital para construir un grupo comprometido para la realización de un proyecto colaborativo, y promueve una actitud integradora, el desarrollo de estrategias flexibles y adaptativas, y ha demostrado ser exitoso en evitar conflictos en las primeras etapas del desarrollo de un proyecto grupal, detectando en forma temprana problemas y la capacidad de priorizar posibles soluciones, en una lógica de triple beneficio (gano yo, gana mi comunidad y, gana el planeta tierra).

La metodología Art of Hosting enseña a las personas a dirigir conversaciones que parten del entendimiento mutuo y trabajan hacia la planificación colectiva; y con esto, mejorar la participación social y las oportunidades de aprendizaje colaborativo. El método que puede implementarse con un gran número de estudiantes (Quick y Sandfort, 2014). El World Café es una metodología que facilita un proceso de conversación, que ayuda a las personas a entablar un diálogo para construir relaciones personales, fomentar el aprendizaje colaborativo y descubrir nuevas posibilidades de acción; facilitando la participación de grandes grupos (de 15 a 100 personas o más) que pueden colaborar de manera creativa, como parte de una sola conversación conectada (Chang y Chen, 2015). 
En el caso de los académicos, el interés del CEM ha sido trasmitir estas nuevas metodologías para facilitar aprendizajes significativos en los estudiantes y desarrollar nuevas formas de evaluación con métodos activos, que desde la perspectiva del programa, son de suma importancia para acercar al mundo académico de áreas alejadas de la ciencias empresariales y de negocios, en la cultura del emprendimiento y generación de ideas de negocios; lo cual facilita un mayor alcance del programa y mayor impacto en cuanto a número de estudiantes que pueden participar en la asignatura. En la figura 1 se puede ver un modelo conceptual del CEM, que considera las acciones que habitualmente desarrolla el programa para fortalecer la vinculación con el medio, y las acciones relacionadas con una formación más integral de los estudiantes de la Universidad.

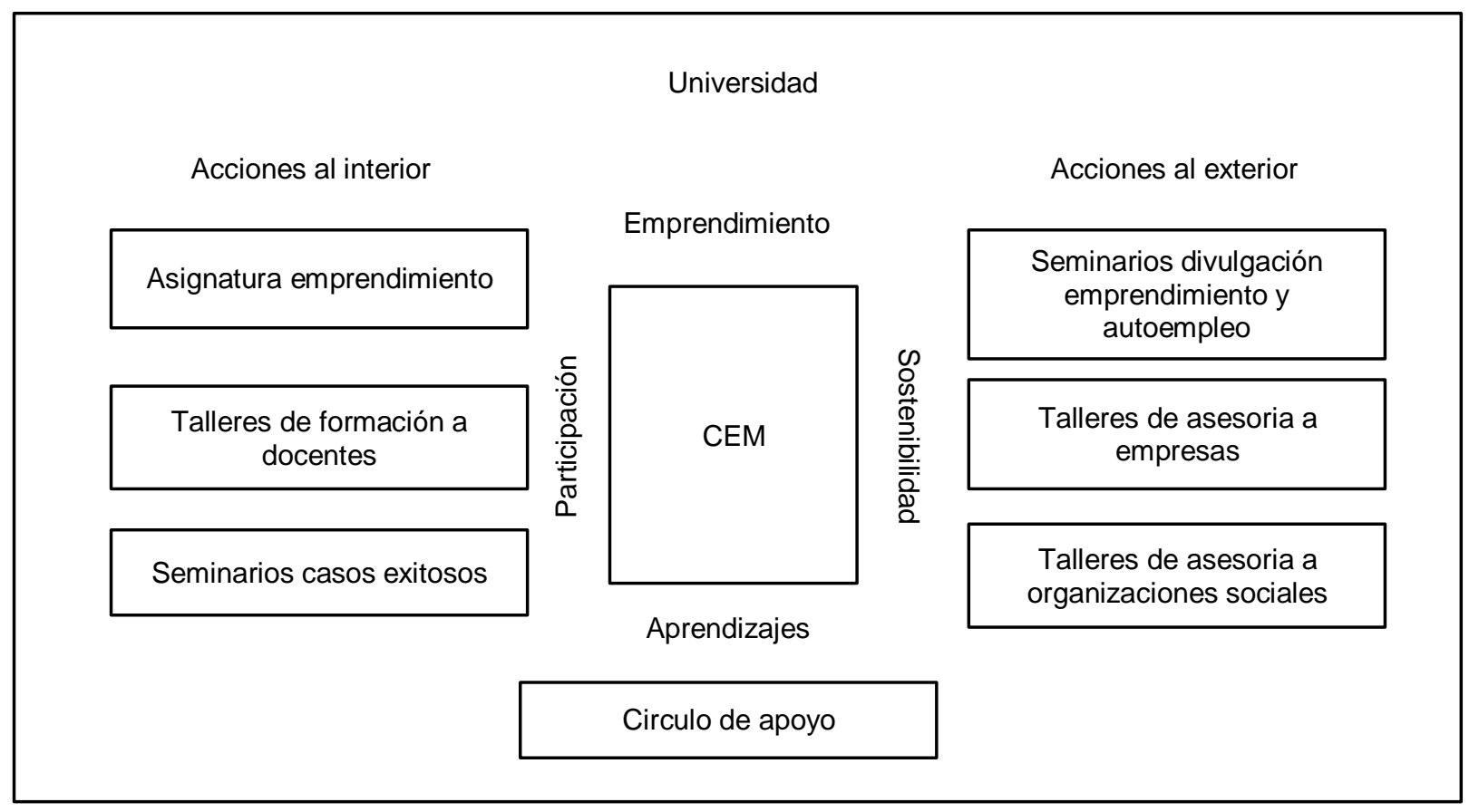

Fig. 1: Modelo Integrado de acciones y objetivos del CEM.

De las acciones realizadas hacia el exterior o denominado habitualmente como vinculación con el medio, se realizan seminarios de divulgación de forma periódica y tienen el fin de reflexionar en torno a los desafíos regionales o nacionales que afectan a la comunidad local; además se realizan escuelas de verano en donde se desarrollan temáticas de interés (adultos mayores, inmigrantes, jóvenes, industria 4.0, automatización y desempleo) vinculados al empleo y emprendimiento. También se contabilizan como acciones al exterior, los talleres de asesorías a empresas y emprendedores (no formalizado), que se realizan en colaboración con los alumnos de último año de la carrera de ingeniería comercial, en donde se presta asesoría en forma gratuita durante un semestre, en temas de diseño de estrategias de comercialización, planificación y mejora de la gestión del negocio a las empresas formales; y en el caso de emprendedores informales, en estrategias de formalización, y diseño del plan de negocio, entre otros temas. También como acciones complementarias para fortalecer el vinculo con el medio, se han realizado talleres de asesorías a organizaciones sociales en formulación de proyectos comunitarios, con enfoque en el desarrollo local y problemáticas ambientales, en donde habitualmente se deben complementar con evaluaciones de la rentabilidad económica y social, que este tipo de proyectos tiene para que sean aprobados o admitidos como admisibles, y que las organizaciones sociales dada su condición de vulnerabilidad, no cuentan con los recursos económicos para disponer de asesorías en este tipo de temas.

En el caso de las acciones al interior de la Universidad, se puede mencionar la asignatura de "Emprendizaje colaborativo", curso optativo que se oferta con cupos limitados a todas las carreras de la Universidad, y en donde utilizando las metodologías mencionadas anteriormente, se desarrollan ideas de negocios en grupos de alumnos que son de diferentes carreras y facultades, generando procesos de aprendizaje interdisciplinario. También se han realizado talleres de formación a docentes en las metodologías usadas en el CEM como alternativas para el desarrollo de aprendizajes significativos y herramientas para el trabajo grupal. Además, se han desarrollado seminarios de casos exitosos en donde se selecciona aquellos grupos que han podido fortalecer una idea de negocio con viabilidad, para exponerlos ante la comunidad, en donde se invita a instituciones públicas y empresarios, para conocer y comentar los proyectos trabajados por los alumnos, en miras de posibles fuentes de financiamiento, mejora de las ideas o planes de negocios, asesoría a la protección de la propiedad intelectual y retroalimentación de la experiencia de los empresarios participantes. 
Respecto de la forma de financiamiento que tiene el Programa, se puede comentar que es de origen mixto, donde la Universidad a través de la Facultad, facilitan un espacio que está dedicado en forma exclusiva para esta iniciativa, el cual cuenta con salas para la docencia, espacios de trabajo y sala de reuniones; y académicos que se vinculan con los diferentes proyectos que se realizan en el CEM. Los profesionales de apoyo y la dirección ejecutiva se financian vía proyectos concursables externos, que facilitan contar con una organización flexible, en donde las directrices generales de las iniciativas y los proyectos son coordinadas entre la directora del CEM y el Consejo de la Facultad; y las decisiones de gestión interna son administradas por una directora ejecutiva.

\section{RESULTADOS}

El programa "Centro de Emprendizaje" de la Universidad Austral de Chile ha aportado a fortalecer y profundizar los vínculos con el medio y los procesos de aprendizaje de los estudiantes que han tenido interés en desarrollar el espíritu emprendedor. Además, ha posibilitado ampliar la red de colaboración de nivel nacional e internacional de la Universidad, a través de la articulación de proyectos asociativos entre diferentes universidades e instituciones públicas, y facilitado el trabajo colaborativo a nivel internacional, accediendo a redes de cooperación internacional como el Community Innovators Lab (CoLab) en MIT o The Talloires Network; y el intercambio de estudiantes y profesores en el marco del programa Erasmus+ de la Unión Europea, permitido que los profesionales del CEM hayan compartido el trabajo desarrollado en la Universidad en otras universidades del mundo, y aprendan formas innovadoras de enseñar y poner a disposición sus saberes desde un contexto de educación no formal.

Las acciones de vinculación implementada por el CEM han aportado a que la Facultad desarrolle relaciones duraderas con la comunidad; por mencionar un ejemplo concreto, un estudiante de la UACh de una comunidad indígena en Paillaco (comuna de la región de Los Ríos) se involucró en uno de los cursos de pregrado del CEM. Como resultado, el alumno contactó al Municipio local para que se puedan replicar los talleres en la comunidad y generar ideas colectivas que mejoren la calidad de vida de los habitantes de la comuna. Estos talleres a su vez motivaron a estudiantes de secundaria, representantes de asociaciones de vecinos, cooperativas y personal municipal a cargo de iniciativas de desarrollo local a la creación de la Oficina de la Juventud en el municipio local y a la Cooperativa de Jóvenes Profesionales de Paillaco. Estas iniciativas no fueron sugeridas por el CEM, pero surgieron de las conversaciones que facilitó en la comunidad. Más tarde, el CEM se asoció con la Unidad Municipal para el Desarrollo Comunal, la Oficina para la Juventud, las organizaciones comunitarias y otros líderes locales para pensar en cómo apoyar pequeñas empresas que podrían impulsar las oportunidades de generación de ingresos para los residentes de Paillaco, con un enfoque particular en las mujeres y los jóvenes. EI CEM también ha aportado a la internacionalización de la Universidad a través de participar en dos redes internacionales con participantes de más de 16 países, en cuatro continentes. El programa YEPI de la red The Talloires Network (https://talloiresnetwork.tufts.edu/the-youtheconomic-participation-initiative/) y el programa RED 2.0, una red de organizaciones comprometidas en 10 países que promueven el voluntariado y el autoempleo para jóvenes socialmente desfavorecidos, con sede en Portugal.

En la tabla 1 se puede ver un resumen estadístico de la cantidad de estudiantes involucrados con el CEM en el período 2015(1)-2018(1) en donde se puede ver que el programa ha logrado formar a más de 355 estudiantes, impulsado más de 39 proyectos colaborativos, que involucraron de 6 a 25 carreras diferentes, lo que refleja el importante grado interdisciplinar logrado en los proyectos. De la tabla 1 se puede ver, que a pesar de ser un curso optativo y de enfoque transversal, ha captado el interés de los estudiantes en forma sostenida en el tiempo, involucrando una diversidad de carreras y facultades, y permitido la realización de proyectos de emprendimiento colaborativos o de proyectos sociales con impacto en las economías locales.

Tabla 1: Número de participantes del curso optativo de "Emprendizaje colaborativo" de la universidad.

\begin{tabular}{|l|c|c|c|c|c|c|}
\hline Año & Semestre & Total alumnos & Hombres & Mujeres & Carreras & Proyectos \\
\hline 2015 & 1 & 43 & 29 & 14 & 25 & 6 \\
\hline 2015 & 2 & 40 & 23 & 17 & 9 & 7 \\
\hline 2016 & 1 & 58 & 32 & 26 & 10 & 8 \\
\hline 2016 & 2 & 65 & 26 & 39 & 9 & 7 \\
\hline 2017 & 1 & 44 & 25 & 19 & 11 & 4 \\
\hline 2017 & 2 & 57 & 32 & 25 & 6 & 3 \\
\hline 2018 & 1 & 48 & 28 & 20 & 12 & 4 \\
\hline Total & & 355 & 195 & 160 & & 39 \\
\hline
\end{tabular}




\section{DISCUSIÓN FINAL}

Hoy en día la sociedad exige nuevas tareas a las universidades, que sea capaz de formar a los estudiantes más allá de una formación tradicional enfocada en la transmisión de conocimiento, hacia una en la que el estudiante desarrolle habilidades y competencias para la vida laboral, y si lo desea pueda emprender nuevos negocios. En este nuevo contexto, la formación en emprendimiento juega un rol clave en estimular el espíritu emprendedor, y debe ser asumida por las universidades en su currículo formativo. Sin embargo, esta situación plantea diversos desafíos a las universidades en términos de definir un programa formativo y las competencias vinculadas al desarrollo de nuevos negocios; además de definir quién y cómo se desarrollarán estas labores formativas al interior de la universidad. En el caso de Chile existen antecedentes que mencionan que las universidades están mostrando una preocupación por asumir esta nueva tarea; y existen diversas iniciativas, que presentan una heterogeneidad en cuanto a su grado de desarrollo y resultados esperados, con lo cual es un campo de desarrollo por parte de las universidades y sus académicos.

El programa CEM implementado por la Universidad Austral de Chile, es una de las diversas experiencias recientes en el ámbito de la educación superior de Chile, y aunque la mayoría de las universidades tienen incubadoras y centros que asesoran a las empresas, no hay muchas iniciativas que promuevan la cultura del emprendimiento, por lo que conocer este tipo de experiencias puede enriquecer el desarrollo de esta tarea al interior de las universidades. El programa, en un enfoque que combina los conceptos de emprender y aprender como un círculo virtuoso, y ha desarrollado una serie de iniciativas que tienen como objetivo el fomentar el emprendimiento en la Facultad de Cs. Económicas y Administrativas y en otras facultades de la Universidad.

De los resultados que hasta el momento ha logrado el programa CEM, se puede mencionar la creación de una asignatura facultativa de pregrado, que cualquier estudiante de la Universidad pueda inscribir, y que mediante metodologías lúdicas y participativas, desarrolla habilidades y competencias para la creación de proyectos en colaboración y multidisciplinarios, que estimulan el emprendimiento y las competencias sello de la Universidad. Esta asignatura se ha dictado de forma permanente en estos últimos años gracias a la flexibilidad institucional en los diferentes niveles de la Universidad, como se recomienda en la literatura (Zaharia y Gibert, 2005) y gracias al financiamiento de diversas fuentes públicas de nivel nacionales e internacional. También como resultados se puede mencionar la constante generación de espacios de encuentro entre la comunidad docente, los estudiantes, los empresarios, emprendedores informales y las organizaciones sociales, que fortalece los vínculos en el interior de la organización y entre la Universidad y su medio. Como resultado inesperado, el trabajo realizado por el programa ha fortalecido la vinculación con el medio de la universidad a nivel nacional e internacional, a través de la participación del programa en redes nacionales e internacionales vinculadas al emprendimiento, que ha posibilitado el intercambio de estudiantes y académicos, y la generación de proyectos de colaboración internacional.

\section{CONCLUSIONES}

De acuerdo al trabajo presentado, a los resultados obtenidos y su discusión, se pueden plantear las siguientes conclusiones principales:

1.- Las universidades tienen el desafío muy reciente de fomentar el emprendimiento entre sus estudiantes de pregrado y académicos, para dar respuesta a los actuales requerimientos de la sociedad y las empresas, y que tienen relación con que los estudiante desarrollen habilidades y competencias para la vida laboral y para emprender nuevos negocios.

2.-En este contexto chileno, la enseñanza del emprendimiento es un tema muy reciente, que requiere de desarrollo y maduración, existiendo diferentes experiencias y métodos para abordar la temática.

3.- La Universidad Austral de Chile ha logrado implementar un programa que busca fomentar el emprendimiento y el trabajo colaborativo e interdisciplinario entre sus estudiantes y docentes a través de diferentes iniciativas, que mediante métodos lúdicos y participativos, ha logrando implementar diferentes iniciativas en los últimos años. Esta experiencia puede aportar a la discusión y desarrollo de este tipo de programas en las universidades chilenas.

\section{REFERENCIAS}

Audretsch, D. B., From the Entrepreneurial University to the University for the Entrepreneurial Society, doi: 10.1007/s10961-012-9288-1, Journal of Technology Transfer, 39(3), 313-321 (2014).

Chang, W. L., y Chen, S. T., The impact of World Café on Entrepreneurial Strategic Planning Capability, doi: 10.1016/j.jbusres.2014.11.020, Journal of Business Research, 68(6), 1283-1290 (2015). 
Clark, B. R., The Entrepreneurial University: Demand and Response, doi: doi.org/10.1007/BF02679392, Tertiary Education and Management, 4(1), 5-16 (1998).

Clark, B. R., Delineating the Character of the Entrepreneurial University, doi: 10.1057/palgrave.hep.8300062, Higher Education Policy, 17(4), 355-370 (2004).

Disterheft, A., Azeiteiro, U. M., Leal Filho, W., y Caeiro, S., Participatory Processes in Sustainable Universities - What to Assess?, doi: 10.1108/IJSHE-05-2014-0079, International Journal of Sustainability in Higher Education, 16(5), 748-771 (2015)

Disterheft, A., Caeiro, S. S., Leal Filho, W., y Azeiteiro, U. M., The INDICARE-Model - Measuring and Caring About Participation in Higher Education's Sustainability Assessment, doi: 10.1016/J.ECOLIND.2015.11.057, Ecological Indicators, 63, 172-186 (2016).

Etzkowitz, H., The Evolution of the Entrepreneurial University, doi: 10.1504/IJTG.2004.004551, International Journal of Technology and Globalisation, 1(1), 64-77 (2004).

Etzkowitz, H., Anatomy of the Entrepreneurial University, doi: 10.1177/0539018413485832, Social Science Information, 52(3), 486-511 (2013).

Etzkowitz, H., Webster, A., Gebhardt, C., y Terra, B. R. C., The Future of the University and the University of the Future: Evolution of Ivory Tower to Entrepreneurial Paradigm, doi: 10.1016/S0048-7333(99)00069-4, Research Policy, 29(2), 313330 (2000).

Etzkowitz, H., y Zhou, C., Building the Entrepreneurial University: A Global Perspective, doi: 10.3152/030234208X363178, Science and Public Policy, 35(9), 627-635 (2008).

Freel, M., Persaud, A., y Chamberlin, T., Faculty Ideals and Universities' Third Mission, doi: 10.1016/J.TECHFORE.2019.06.019, Technological Forecasting and Social Change, 147, 10-21 (2019).

Gibb, A., Haskins, G., y Robertson, I., Leading the Entrepreneurial University: Meeting the Entrepreneurial Development Needs of Higher Education Institutions, in Universities in Change: Innovation, Technology, and Knowledge Management, pp 9-45, Springer, New York, USA (2013).

Grigg, T., Adopting an Entrepreneurial Approach in Universities, doi: 10.1016/0923-4748(94)90013-2, Journal of Engineering and Technology Management, 11(3-4), 273-298 (1994).

Heaton, S., Siegel, D. S., y Teece, D. J., Universities and Innovation Ecosystems: A Dynamic Capabilities Perspective, doi: 10.1093/icc/dtz038, Industrial and Corporate Change, 28(4), 921-939 (2019).

Herrera, R. A. y Villalobos, M. A., Caracterización de la Formación Académica en Emprendimiento de Pregrado que se realiza en Universidades Chilenas, doi: 10.4067/S0718-50062018000400043, Formación Universitaria, 11(4), 43-52 (2018).

Lazzeroni, M., y Piccaluga, A., Towards the Entrepreneurial University, doi: 10.1080/0269094032000073807, Local Economy, 18(1), 38-48 (2003).

Mandakovic, V., Cohen, B., y Amorós, J. E., Entrepreneurship Policy and its Impact on the Cultural Legitimacy for Entrepreneurship in a Developing Country Context, in Entrepreneurship, Regional Development and Culture: An Institutional Perspective, pp 109-125, Springer, Cham, Switzerland (2015).

Martinelli, A., Meyer, M., y Von Tunzelmann, N., Becoming an Entrepreneurial University? A case Study of Knowledge Exchange Relationships and Faculty Attitudes in a Medium-Sized, Research-Oriented University, doi: 10.1007/s10961007-9031-5, The Journal of Technology Transfer, 33(3), 259-283 (2008).

Marzocchi, C., Kitagawa, F., y Sánchez-Barrioluengo, M., Evolving Missions and University Entrepreneurship: Academic Spin-offs and Graduate Start-ups in the Entrepreneurial Society, doi: 10.1007/s10961-017-9619-3, The Journal of Technology Transfer, 44(1), 167-188 (2019).

Mondaca, C., Lopatinsky, J., Montecinos, A. M., y Rojas-Mora, J., Medición del Nivel de Desarrollo de las Universidades Chilenas: Un Análisis con Modelos de Ecuaciones Estructurales, doi: 10.31619/caledu.n50.562, Calidad en La Educación, 50, 284-318 (2019).

Quick, K., y Sandfort, J., Learning to Facilitate Deliberation: Practicing the Art of Hosting, doi: 10.1080/19460171.2014.912959, Critical Policy Studies, 8(3), 300-322 (2014).

Scharmer, C. O., Theory U: Learning From the Future as it Emerges, 2da ed., pp 544. Berrett-Koehler Publishers, San Francisco, California, USA (2016).

Smilor, R. W., Dietrich, G. B., y Gibson, D. V., The entrepreneurial University: the Role of Higher Education in the United States in Technology Commercialization and Economic Development, International Social Science Journal, ISSN: 00208701, 45(135), 1-11 (1993).

Soria-Barreto, K., Zuniga-Jara, S., y Ruiz-Campo, S., Educación e Intención Emprendedora en Estudiantes Universitarios: Un Caso de Estudio, doi: 10.4067/S0718-50062016000100004, Formación Universitaria, 9(1), 25-34 (2016).

UACh., Competencias sello UACh (en linea), https://www.uach.cl/uach/ file/competencias-sello-uach.pdf. Acceso: 30 de Marzo (2020). 
Wong, P.-K., Ho, Y.-P., y Singh, A., Towards an "Entrepreneurial University" Model to Support Knowledge-Based Economic Development: The Case of the National University of Singapore, doi: 10.1016/j.worlddev.2006.05.007, World Development, 35(6), 941-958 (2007).

Zaharia, S. E., y Gibert, E., The Entrepreneurial University in the Knowledge Society, doi: 10.1080/03797720500088038, Higher Education in Europe, 30(1), 31-40 (2005).

Zhang, Y., Duysters, G., y Cloodt, M., The Role of Entrepreneurship Education as a Predictor of University Students' Entrepreneurial Intention, doi: 10.1007/s11365-012-0246-Z, International Entrepreneurship and Management Journal, 10(3), 623-641 (2014) 